\title{
Exact treatment of linear difference equations with noncommutative coefficients
}

\author{
M. A. Jivulescu, A. Messina, A. Napoli \\ MIUR, CNISM and Dipartimento di Scienze Fisiche ed Astronomiche, \\ Università di Palermo, via Archirafi 36, 90123 Palermo, Italy \\ F. Petruccione
}

School of Physics, University of KwaZulu-Natal, Private Bag X54001, Durban 4000, South Africa

\begin{abstract}
SUMMARY
The exact solution of a Cauchy problem related to a linear second-order difference equation with constant noncommutative coefficients is reported.
\end{abstract}

\section{INTRODUCTION}

Difference equations are ubiquitous in applied sciences. The mathematical theory underlying the treatment of these equations is well consolidated and explicit systematic methods for solving special classes of difference equations are well known. For instance the key to get the general solution of a linear difference equation with constant coefficients, as for linear differential equations once again with constant coefficients, is to find generally the complex roots of an algebraic equation known as the characteristic equation. To write down the general solution at this point is only a trivial systematic matter.

The simplicity of the second-order difference equation

$$
Y_{p+2}=\mathcal{L}_{0} Y_{p}+\mathcal{L}_{1} Y_{p+1}, \quad p=0,1,2, \ldots=\mathbb{N}
$$

is, however, only apparent when $\mathcal{L}_{0}, \mathcal{L}_{1}$, supposed independent from $p$ and then behaving as constant coefficients, are noncommutative "mathematical objects". The specific nature of these coefficients, as well as of $Y_{p}$ and of the same "operations" appearing in equation (1) is of course strictly related with the "abstract support space" in which the scientific problem represented by the equation under scrutiny is formulated. Thus, for example, let our reference space be an $n$-dimensional linear vector space $M$, then $Y_{p}$ is a vector belonging to $M$, while $\mathcal{L}_{0}, \mathcal{L}_{1}$ are linear operators acting upon the vectors of $M$. Equation (1) may even represent a matrix equation, interpreting both the unknowns $Y_{p}$ and the coefficients $\mathcal{L}_{0}, \mathcal{L}_{1}$ as matrices of given order[1]. 
If $x \in \mathbb{R}$ and $Y_{p} \equiv f_{p}(x)$ is a $C^{\infty}$ - function, then $\mathcal{L}_{0}, \mathcal{L}_{1}$ might be noncommutative operators involving for example derivatives of any order.

In the context of quantum mechanics the unknowns of equation (1) might be elements of a sequence of operators belonging to the Hilbert vector space of the physical system and $\mathcal{L}_{0}, \mathcal{L}_{1}$ appropriate noncommutative superoperators $[2,3]$.

These simple examples are sufficient to motivate the interest toward the search of techniques for solving equation (1) when $\mathcal{L}_{0}, \mathcal{L}_{1}$ are noncommutative coefficients. In this paper we cope with a Cauchy problem associated with equation (1) and succeed in giving its explicit solution independently on the scientific context in which equation (1) has been formulated.

We do not choose from the very beginning the mathematical nature of its ingredients, rather we only require that all the symbols and operations appearing in equation (1) are meaningful. Thus "vectors" $Y$ may be added, this operation being commutative and at the same time may be acted upon by $\mathcal{L}_{0}$ or $\mathcal{L}_{1}$ ( hereafter called operators) transforming themselves into other "vectors".

The symbol $Y_{0}=0$ simply denotes, as usual, the neutral elements of the addition between vectors. Finally we put $\left(\mathcal{L}_{a} \mathcal{L}_{b}\right) Y \equiv \mathcal{L}_{a}\left(\mathcal{L}_{b} Y\right) \equiv \mathcal{L}_{a} \mathcal{L}_{b} Y$ with $a$ or $b=0,1$ and define addition between operators through linearity.

The paper is organized as follows.

Some mathematical preliminaries open the next section in which the Cauchy problem associated with equation (1) is formulated. In the same section we state and demonstrate our main result which is the formula for solution of the Cauchy problem. In the subsequent section we show the reduction of our formula to the case of commutative coefficients by discussing a specific example. Some concluding remarks are presented in the last section.

\section{THE CAUCHY PROBLEM: DEFINITION AND RESOLUTION}

We start by introducing some mathematical preliminaries.

Let $u \in \mathbb{N}$ and $v \in \mathbb{N}$ and consider the couple of generally noncommutative coefficients $\mathcal{L}_{0}$ and $\mathcal{L}_{1}$ appearing in equation (1).

We introduce the symbol $\left\{\mathcal{L}_{0}^{(u)} \mathcal{L}_{1}^{(v)}\right\}$ to denote the sum of all possible distinct permutations of $u$ factors $\mathcal{L}_{0}$ and $v$ factors $\mathcal{L}_{1}$. A few examples help to illustrate the notation:

$$
\begin{gathered}
\left\{\mathcal{L}_{0}^{(0)} \mathcal{L}_{1}^{(0)}\right\}=I,\left\{\mathcal{L}_{0}^{(0)} \mathcal{L}_{1}^{(1)}\right\}=\mathcal{L}_{1} \\
\left\{\mathcal{L}_{0}^{(1)} \mathcal{L}_{1}^{(1)}\right\}=\mathcal{L}_{0} \mathcal{L}_{1}+\mathcal{L}_{1} \mathcal{L}_{0}
\end{gathered}
$$




$$
\left\{\mathcal{L}_{0}^{(1)} \mathcal{L}_{1}^{(2)}\right\}=\mathcal{L}_{0} \mathcal{L}_{1}^{2}+\mathcal{L}_{1}^{2} \mathcal{L}_{0}+\mathcal{L}_{1} \mathcal{L}_{0} \mathcal{L}_{1}
$$

When we exploit combinatorial theory, it is not difficult to convince ourselves that the number of all the possible different terms appearing in $\left\{\mathcal{L}_{0}^{(u)} \mathcal{L}_{1}^{(v)}\right\}$ coincides with the binomial coefficient $\left(\begin{array}{c}u+v \\ m\end{array}\right), m$ being the minimum between $u$ and $v$ that is $m=\min (u, v)$.

We now consider the Cauchy problem

$$
\left\{\begin{array}{c}
Y_{p+2}=\mathcal{L}_{0} Y_{p}+\mathcal{L}_{1} Y_{p+1} \\
Y_{0}=0, Y_{1}=\bar{Y}_{1}
\end{array}, \quad p \in \mathbb{N},\right.
$$

with $\mathcal{L}_{0}$ and $\mathcal{L}_{1}$ in general noncommutative coefficients.

Then we state the following

Theorem 1 The solution of the Cauchy problem (2) can be written as

$$
Y_{p}=\sum_{t=0}^{\bar{t}_{p}}\left\{\mathcal{L}_{0}^{(t)} \mathcal{L}_{1}^{(p-1-2 t)}\right\} \bar{Y}_{1}
$$

where

$$
\bar{t}_{p}=\left[\frac{p-1}{2}\right]=\left\{\begin{array}{cccc}
\frac{p-2}{2}, & \text { if } & p & \text { even } \\
\frac{p-1}{2}, & \text { if } & p & \text { odd }
\end{array},\right.
$$

denoting by $[x]$ the integer part of $x \in \mathbb{R}$.

Proof:

In order to prove that equation (3) gives the solution of the Cauchy problem (2) we exploit the procedure of mathematical induction.

To this end we put $p=1$ in equation (3). It is immediate to verify that, in this case, equation (3) verifies the initial condition $Y_{1}=\bar{Y}_{1}$.

We now consider the case $p=2$. The sum appearing in equation (3) reduces, in this case, to a single term corresponding to $t=0$. We have indeed $Y_{2}=\mathcal{L}_{1}\left(\bar{Y}_{1}\right)$. Once again it is easy to convince ourselves that $Y_{2}$ satisfies equation (2).

We now suppose that the first $(p+1)$ terms of the sequence solution may be represented by equation (3). We have to prove that $Y_{p+2}$ also can be expressed by equation (3).

We observe that by the inductive hypothesis

$$
\begin{array}{r}
\mathcal{L}_{0} Y_{p}+\mathcal{L}_{1} Y_{p+1}=\sum_{t=0}^{\bar{t}_{p}} \mathcal{L}_{0}\left\{\mathcal{L}_{0}^{(t)} \mathcal{L}_{1}^{(p-1-2 t)}\right\} \bar{Y}_{1}+\sum_{t=0}^{\bar{t}_{p+1}} \mathcal{L}_{1}\left\{\mathcal{L}_{0}^{(t)} \mathcal{L}_{1}^{(p-2 t)}\right\} \bar{Y}_{1}= \\
\sum_{t=0}^{\bar{t}_{p}} \mathcal{L}_{0}\left\{\mathcal{L}_{0}^{(t)} \mathcal{L}_{1}^{(p-1-2 t)}\right\} \bar{Y}_{1}+\sum_{t=1}^{\bar{t}_{p+1}} \mathcal{L}_{1}\left\{\mathcal{L}_{0}^{(t)} \mathcal{L}_{1}^{(p-2 t)}\right\} \bar{Y}_{1}+\mathcal{L}_{1}^{(p+1)} \bar{Y}_{1}
\end{array}
$$


It is not difficult to see that equation (4) implies

$$
\bar{t}_{p+1}=\left\{\begin{array}{c}
\bar{t}_{p}+1, \quad \text { if } p \text { even } \\
\bar{t}_{p}, \quad \text { if } p \text { odd }
\end{array} .\right.
$$

The right member of the equation (5) can be thus rewritten as

$$
\begin{aligned}
& \mathcal{L}_{1}^{(p+1)} \bar{Y}_{1}+\mathcal{L}_{0}^{(1)} \mathcal{L}_{1}^{(p-1)} \bar{Y}_{1}+\mathcal{L}_{1}\left\{\mathcal{L}_{0}^{(1)} \mathcal{L}_{1}^{(p-2)}\right\} \bar{Y}_{1}+\mathcal{L}_{0}\left\{\mathcal{L}_{0}^{(1)} \mathcal{L}_{1}^{(p-3)}\right\} \bar{Y}_{1}+ \\
& \mathcal{L}_{1}\left\{\mathcal{L}_{0}^{(2)} \mathcal{L}_{1}^{(p-4)}\right\} \bar{Y}_{1}+\cdots+\mathcal{L}_{0}\left\{\mathcal{L}_{0}^{(t)} \mathcal{L}_{1}^{(p-1-2 t)}\right\} \bar{Y}_{1}+\mathcal{L}_{1}\left\{\mathcal{L}_{0}^{(t+1)} \mathcal{L}_{1}^{(p-2(t+1))}\right\} \bar{Y}_{1}+ \\
& \cdots+\left\{\begin{array}{r}
\mathcal{L}_{0}\left\{\mathcal{L}_{0}^{\left(\bar{t}_{p}\right)} \mathcal{L}_{1}^{\left(p-1-2 \bar{t}_{p}-1\right)}\right\} \bar{Y}_{1}+\mathcal{L}_{1}\left\{\mathcal{L}_{0}^{\left(\bar{t}_{p}+1\right)} \mathcal{L}_{1}^{\left(p-2\left(\bar{t}_{p}+1\right)\right)}\right\} \bar{Y}_{1}, \quad \text { if } \quad p \quad \text { even } \\
\mathcal{L}_{0}\left\{\mathcal{L}_{0}^{\left(\bar{t}_{p}\right)} \mathcal{L}_{1}^{\left(p-1-2 \bar{t}_{p}\right)}\right\} \bar{Y}_{1}=\mathcal{L}_{0}^{\frac{p+1}{2}} \bar{Y}_{1}, \quad \text { if } \quad p \quad \text { odd }
\end{array}\right.
\end{aligned}
$$

Concentrating now on the general $t^{t h}$ - operator term of equation (7), that is

$$
\mathcal{L}_{0}\left\{\mathcal{L}_{0}^{(t)} \mathcal{L}_{1}^{(p-1-2 t)}\right\}+\mathcal{L}_{1}\left\{\mathcal{L}_{0}^{(t+1)} \mathcal{L}_{1}^{(p-2(t+1))}\right\}, \quad t \in\left[0, \bar{t}_{p}\right) \cap \mathbb{N}
$$

it is not difficult to persuade ourselves that by definition it expresses the sum of $\left(\begin{array}{c}p-t-1 \\ m\end{array}\right)+\left(\begin{array}{c}p-t-1 \\ m^{\prime}\end{array}\right)$ terms, where $m=\min (t, p-2 t-1)$ and $m^{\prime}=\min (t+1, p-2 t-2)$.

It is possible to verify that, when $t \in\left[0, \bar{t}_{p}\right) \cap \mathbb{N}$, then $m \geq 0, m^{\prime} \geq 0$ and $\left|m-m^{\prime}\right|=1$ so that in according with the well-known Stifel formula

$$
\left(\begin{array}{c}
p-t-1 \\
m
\end{array}\right)+\left(\begin{array}{c}
p-t-1 \\
m^{\prime}
\end{array}\right)=\left(\begin{array}{c}
p-t \\
M
\end{array}\right)
$$

where $M=\max \left(m, m^{\prime}\right)=\min (t+1,(p+2)-1-2(t+1))$.

In view of relation (9) we may thus say that the expression (8) coincides with the sum of all possible distinct permutations of $(t+1)$ factors $\mathcal{L}_{0}$ and $\left[(p+2)-1-2((t+1)]=p-1-2 t\right.$ factors $\mathcal{L}_{1}$. Thus by definition we may legitimately write that

$$
\mathcal{L}_{0}\left\{\mathcal{L}_{0}^{(t)} \mathcal{L}_{1}^{(p-1-2 t)}\right\}+\mathcal{L}_{1}\left\{\mathcal{L}_{0}^{(t+1)} \mathcal{L}_{1}^{(p-2(t+1))}\right\}=\left\{\mathcal{L}_{0}^{(t+1)} \mathcal{L}_{1}^{(p+2)-1-2(t+1)}\right\},(\forall) t \neq \bar{t}_{p}
$$

We also observe that the first term of equation (7) can be cast in the following form

$$
\mathcal{L}_{1}^{p+1}=\mathcal{L}_{0}^{0} \mathcal{L}_{1}^{(p+2)-1-2 \cdot 0}
$$


The definition of $\bar{t}_{p}$ given by equation (4) on the other hand enables us to say that

$$
\bar{t}_{p+2}=\left[\frac{p+1}{2}\right]=\left\{\begin{array}{cccc}
\bar{t}_{p}+1=\frac{p}{2}, & \text { if } & p & \text { even } \\
\bar{t}_{p}+1=\frac{p+1}{2}, & \text { if } & p & \text { odd }
\end{array} .\right.
$$

Thus the last term of expression (7) may be written as

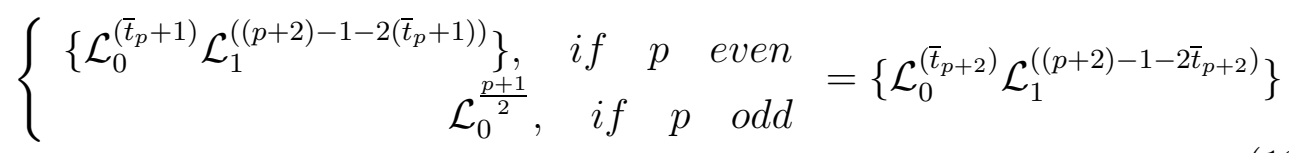

Using relations (7),(10),(11) and (13) we may thus conclude that

$$
\mathcal{L}_{0} Y_{p}+\mathcal{L}_{1} Y_{p+1}=\sum_{t=-1}^{\bar{t}_{p+2}}\left\{\mathcal{L}_{0}^{(t+1)} \mathcal{L}_{1}^{((p+2)-1-2(t+1))}\right\} \bar{Y}_{1}=\sum_{t=0}^{\bar{t}_{p+2}}\left\{\mathcal{L}_{0}^{(t)} \mathcal{L}_{1}^{((p+2)-1-2 t)}\right\} \bar{Y}_{1}
$$

which coincides with $Y_{p+2}$ in accordance with the resolving formula given by equation (3).

\section{REDUCTION TO THE CASE OF COMMUTATIVE COEFFICIENTS}

It is well-known [4] that, when the coefficients $\mathcal{L}_{0}$ and $\mathcal{L}_{1}$ of equation (1) reduce to C-numbers as well as $Y_{p}$, the solution of the relative equation (1) is traced back to the solutions of its characteristic equation. The following theorem summarizes the well known result.

Theorem 2 Consider the equation

$$
y_{p+2}=c_{0} y_{p}+c_{1} y_{p+1},
$$

where $c_{0} \neq 0$ and $c_{1}$ are real constants associated with the initial conditions $y_{0}=1$ and $y_{1}=\bar{y}_{1}$.

If $m_{1}$ and $m_{2}$ are the roots of the characteristic equation

$$
m^{2}-c_{1} m-c_{0}=0, \quad m_{1,2}=\frac{c_{1} \pm \sqrt{\Delta}}{2}, \quad \Delta=c_{1}^{2}+4 c_{0}
$$

then the solution of this Cauchy problem is given by

$$
y_{p}=\left\{\begin{array}{rl}
\frac{1}{m_{1}-m_{2}}\left[m_{1}^{p}-m_{2}^{p}\right] \bar{y}_{1}, & \text { if } \quad \Delta \neq 0 \\
p m_{1}^{p-1} \bar{y}_{1}, & \text { if } \quad \Delta=0
\end{array} .\right.
$$


We now demonstrate that our operator solution (3) reduce to (17) when $\mathcal{L}_{0}=c_{0}$ and $\mathcal{L}_{1}=c_{1}$. We firstly prove the following

Lemma 1 When $\mathcal{L}_{0}=c_{0}$ and $\mathcal{L}_{1}=c_{1}$, then $y_{p}$ given by equation (3) can be reduced to the following expression

$$
y_{p}=\sum_{t=0}^{\bar{t}_{p}}\left\{\mathcal{L}_{0}^{(t)} \mathcal{L}_{1}^{(p-1-2 t)}\right\} \bar{y}_{1}=c_{1}^{p-1} \sum_{t=0}^{\left[\frac{p-1}{2}\right]}\left(\begin{array}{c}
p-t-1 \\
t
\end{array}\right)\left(\frac{c_{0}}{c_{1}^{2}}\right)^{t} \bar{y}_{1}
$$

Proof:

The solution of a linear homogenous second-order difference equation with constant coefficients presented in the form (18) is a consequence of the general result presented in section 2 . In this case the coefficients are constants and so they commute. We may conclude that

$$
y_{p}=\sum_{t=0}^{\bar{t}_{p}}\left(\begin{array}{c}
p-t-1 \\
\min \{t, p-1-2 t\}
\end{array}\right) c_{0}^{t} c_{1}^{p-1-2 t} \bar{y}_{1}
$$

Taking in consideration that

$$
\left(\begin{array}{c}
p-t-1 \\
t
\end{array}\right)=\left(\begin{array}{c}
p-t-1 \\
p-1-2 t
\end{array}\right)
$$

we may rewrite the equation (19) in the simplest way as (18).

In order to cast the expression (18) into the form presented in theorem 2 we consider two cases, accordingly with the nullity or non nullity of the discriminant of the characteristic equation (16).

Assuming $\Delta \neq 0$ we may use the identity $[5,6]$

$$
\sum_{k=0}^{[n / 2]}\left(\begin{array}{c}
n-k \\
k
\end{array}\right) z^{k}=2^{-n-1}(1+4 z)^{-1 / 2}\left[(1+\sqrt{1+4 z})^{n+1}-(1-\sqrt{1+4 z})^{n+1}\right],
$$

where $z \in \mathbb{C}, z=c_{0} / c_{1}^{2}$, to rewrite the equation (18) as

$$
\begin{gathered}
y_{p}=c_{1}^{p-1} 2^{-p}\left(1+\frac{4 c_{0}}{c_{1}^{2}}\right)^{-1 / 2}\left[\left(1+\sqrt{1+\frac{4 c_{0}}{c_{1}^{2}}}\right)^{p}-\left(1-\sqrt{1+\frac{4 c_{0}}{c_{1}^{2}}}\right)^{p}\right] \bar{y}_{1}= \\
\left(\frac{c_{1}}{2}\right)^{p} \frac{1}{\Delta}\left[\left(\frac{c_{1}+\sqrt{\Delta}}{c_{1}}\right)^{p}-\left(\frac{c_{1}-\sqrt{\Delta}}{c_{1}}\right)^{p}\right] \bar{y}_{1}=\frac{1}{\sqrt{\Delta}}\left[\left(\frac{c_{1}+\sqrt{\Delta}}{2}\right)^{p}-\left(\frac{c_{1}-\sqrt{\Delta}}{2}\right)^{p}\right] \bar{y}_{1}= \\
=\frac{1}{m_{1}-m_{2}}\left[m_{1}^{p}-m_{2}^{p}\right] \bar{y}_{1}
\end{gathered}
$$


When on the other hand $\Delta=0$, then $c_{0} / c_{1}^{2}=-1 / 4$ so that exploiting the identity $[5,6]$

$$
\sum_{k=0}^{[n / 2]}\left(-\frac{1}{4}\right)^{k}\left(\begin{array}{c}
n-k \\
k
\end{array}\right)=(n+1) 2^{-n}
$$

we may easily get the following form for equation (18)

$$
y_{p}=\sum_{t=0}^{\left[\bar{t}_{p}\right]}\left(-\frac{1}{4}\right)^{t}\left(\begin{array}{c}
p-1-t \\
t
\end{array}\right) \bar{y}_{1}=p\left(\frac{c_{1}}{2}\right)^{p-1} \bar{y}_{1}=p m_{1}^{p-1} \bar{y}_{1}
$$

\section{CONCLUDING REMARKS}

The main result of this paper is that expressed by equation (3). It provides the exact solution of the Cauchy problem formulated in equation (2). At the best of our knowledge this resolutive formula, the peculiar feature of which is its wide applicability, is a new result demonstrated in this paper for the first time. The importance of being able to treat in a systematic way mathematical problems, wherein effects stemming from noncommutativity cannot be simply overcome, is very easy to appreciate and in addition is not confined to few research areas. For all these reasons we claim that our results are both interesting and useful.

\section{REFERENCES}

1. Gantmacher F.R. The Theory of Matrices American Mathematical Society, Providence, Rhode Island, 1998.

2. Breuer H.-P., Petruccione F. The Theory of Open Quantum Systems Oxford University Press Inc., New York, 2002.

3. Le Bellac M. Quantum Physics Cambridge University Press, Cambridge, 2006.

4. Kelley W. G. Peterson A.C. Difference Equation, Academic Press, London, 2001.

5. Prudnikov A.P. Brychov Yu. A. Marichev O.I. Integrals and Series vol 1, Gordon and Breach Science Publishers, Amsterdam, 1990.

6. Graham R. Knuth D. Patashnik O. Concrete Mathematics, AddisonWesley Publishing Company, Reading, Massachusetts, 1994. 